\title{
INFLUENCE OF INTERFACE-INDUCED DISORDER ON CLASSICAL AND QUANTUM CONDUCTIVITY OF CdTe:IN EPITAXIAL LAYERS
}

\author{
J. Łusakowski, K. Karpierz, M. Grynberg
}

Institute of Experimental Physics, University of Warsaw

Hoża 69, 00-681 Warsaw, Poland

G. Karczewski, T. Wojtowicz

Institute of Physics, Polish Academy of Sciences

Al. Lotników 32/46, 02-668 Warsaw, Poland

\section{S. Contreras and O. Callen}

Groupe d'Etude des Semiconducteurs, Université Montpellier II

Pl. E. Bataillon, Montpellier, France

An influence of disorder originated from the substrate/layer interface on electrical properties of CdTe:In layers was investigated by means of the Hall effect and magnetoresistance measurements at low temperatures. An estimation of a scattering rate due to interface-induced disorder is given. Characteristic features of a magnetic field dependence of magnetoresistance are explained by an influence of quantum interference of scattered electron waves both in the hopping and the free electron conductivity regimes.

PACS numbers: 73.61.Ga, 73.50.Jt

Electrical properties of thin layers are strongly influenced by a substrate/layer interface. The interface is a source of disorder and provides traps for free carriers, which leads to a reduction of the layer's conductivity. In particular, this may happen due to dislocations which appear as a result of a substrate/layer mismatch. In this paper we investigated electrical properties of a doped layer whose distance to the interface was a control parameter.

Investigated structures were MBE grown and consisted of a semi-insulating SI-GaAs substrate on which 3 monolayers of $\mathrm{ZnTe}$, a buffer of nominally undoped $\mathrm{CdTe}$ and a.doped layer were subsequently grown [1]. Samples with a buffer of the thickness ( $d$, the control parameter mentioned above) of $0,0.5,3$ and $7 \mu \mathrm{m}$ and a layer of the thickness of $0.5 \mu \mathrm{m}$ or $1 \mu \mathrm{m}$ were prepared. The nominal doping of all the layers was technologically kept constant and equal to about $5 \times 10^{16} \mathrm{~cm}^{-3}$. 
The measurements were performed after cooling a sample in the dark and were repeated after subsequent illumination and switching off the band-to-band light. The measurements with no illumination were possible only for samples with $d$ equal to 7 or $3 \mu \mathrm{m}$ (hereafter referred to as the samples $A$ and $B$, respectively) since the resistance of other samples cooled in the dark to helium temperatures was higher than $10^{12} \Omega$. The resistance of these samples, even after illumination, was still too high to carry out the Hall effect measurements and in such cases only magnetoresistance data was obtained. In the following we are focused on measurements on the samples $A$ and $B$ with no illumination.

The Hall effect data obtained after cooling a sample in the dark was fitted by a model assuming doping with shallow donors (the concentration $N_{\mathrm{d}}$ ) and conductivity by free electrons only. Scattering processes taken into account in calculating the mobility included scattering by ionised and neutral donors, ionised acceptors and optical and acoustical phonons (deformation potential and piezoelectric mechanisms in the latter case) [2].

Conductivity measurements for the samples $A$ and $B$ as a function of temperature $(T)$ show the hopping conductivity for $T<T_{0} \approx 15 \mathrm{~K}$ and free electrons conductivity for higher $T$. Within the above model, for $T>T_{0}$, one can reproduce the Hall concentration data for the sample $A$ taking $N_{\mathrm{d}}=(5.0 \pm 0.4) \times 10^{16} \mathrm{~cm}^{-3}$ and the compensation $K=0.15 \pm 0.1$. The electron mobility of this sample calculated for the lower (upper) limits of $N_{\mathrm{d}}$ and $K$ is about twice $(50 \%)$ higher than the measured one, but in both cases the shape of the calculated curve differs from that of the measured dependence. Bearing in mind an identical doping of the samples $A$ and $B$ one gets $K=0.80 \pm 0.1$ for the sample $B$.

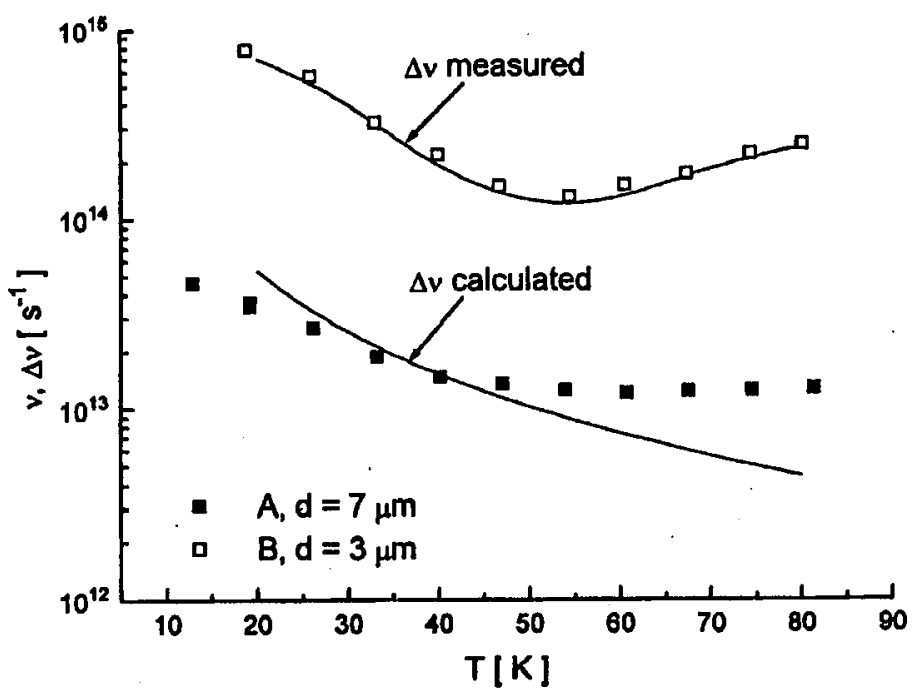

Fig. 1. The measured scattering rates $\nu$ for the samples $A$ (full squares) and $B$ (open squares) with their measured and calculated difference $\Delta \nu$ (the upper and the lower full curve, respectively). 
Results of the Hall mobility $\mu$ measurements are shown in Fig. 1 as the scattering rate $\nu_{A, B}=\mu_{A, B}^{-1} e / m$, where $e$ and $m$ are the electron charge and mass, respectively. The measured difference, $\Delta \nu=\nu_{B}-\nu_{A}$, between the scattering rate for the samples $A$ and $B$ (the upper solid line in Fig. 1) is not reproduced by the model described above (the lower solid line in Fig. 1). The reason of such a large discrepancy is a poor agreement of the model and the experimental data for the sample $B$. In this case the calculated mobility differs by an order of magnitude from the measured one. This shows that the model lacks a scattering mechanism of an effectiveness which is dominant for the sample $B$ but is comparable with an effectiveness of impurity scattering (dominant in the model calculations in the considered range of $T$ ) for the sample $A$. We propose that the lacking scattering is due to interface-induced defects and long-range fluctuations of the electrostatic potential. The source of the fluctuations is a random distribution of charged centres at the interface, in the buffer and the layer itself. Moving the doped layer apart the interface reduces essentially an effectiveness of this scattering which explains why the mobility for the sample $A$ is higher than that for the sample $B$. It is interesting to note that decreasing $d$ by $4 \mu \mathrm{m}$ increases the scattering rate by 10 times and the measured $\nu$ for the sample $B$ (open symbols in Fig. 1) is in fact due to the interface-induced disorder scattering.

For $T<T_{0}$ the temperature dependence of the resistance follows the Mott law of the variable range hopping [3] as was deduced from the temperature dependence of conductivity. An estimate of the electron localization length, $\xi$, and the optimal hopping distance, $R_{\text {hop }}$, gives for both samples about $50 \AA$ and $200 \AA$, respectively. The low-field magnetoresistance shows a minimum at $B_{\min }$ (see the inset in Fig. 1) whose position is constant for $T<T_{0}$ and is equal to $(1.3 \pm 0.1) \mathrm{T}$ and $(0.9 \pm 0.1) \mathrm{T}$ for the sample $A$ and $B$, respectively. For higher temperatures $B_{\min }$ grows with $T$. For $B>3 \mathrm{~T}$ a strong increase in the resistance is observed which is due to a shrinkage of the wave function of electrons hopping between donors [4]. For the sample $A$, the resistance follows $\exp \left(\alpha B^{2}\right)$ dependence, while for the sample $B$ an increase in the resistance is slower than that (Fig. 2). The magnetoresistance is identical for $B$ perpendicular and parallel to the current, in agreement with the theory of hopping conductivity.

The minimum of the magnetoresistance in the hopping regime is often explained as a result of a quantum interference of partial waves of a tunnelling electron which is scattered by ionised centres situated near the tunnelling path [5]. On the other hand, for temperatures corresponding to the conduction by free electrons the minimum originates from weak localization [6], a result of the interference of partial waves of a free electron. The major difference between these two interference phenomena is that in the case of hopping the scattering involved is forward directed and in the case of the weak localization is the backward one. This in turn is a consequence of the exponentially decreasing wave function of a hopping electron which makes negligibly small a contribution of return paths to the interference pattern.

A slower than $\exp \left(\alpha B^{2}\right)$ dependence of the magnetoresistance for the sample $B$ can be explained by a reduction of the magnetic barrier by the scattering events occurring during an electron hop [7]. There is no evidence of such a reduction for 


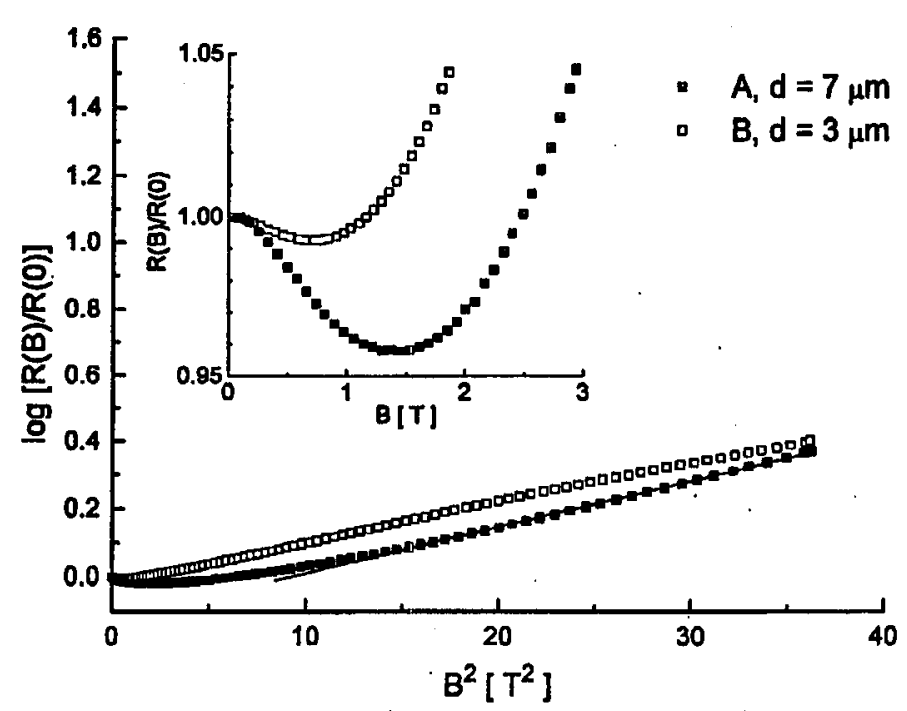

Fig. 2. The magnetoresistance, $R(B) / R(0)$, of the sample $A$ and $B$ (the lower and the upper curve, respectively) as a function of the magnetic field, $B$, for $T=4.2 \mathrm{~K}$. The solid line follows $\exp \left(\alpha B^{2}\right)$ dependence, with $\alpha=0.014$. The inset shows a region of the minimum of magnetoresistance at small magnetic fields.

the sample $A$. This is consistent with a plausible assumption that since $R_{\text {hop }}$ is comparable for both samples an electron during a hop undergoes more scattering events in the sample $B$ than in $A$, just because of a stronger disorder in the latter. It follows then that the stronger sub-barrier scattering, the smaller $B_{\min }$. Thus, in the case of the results shown above, $B_{\min }$ can be used as a measure of disorder. More data on samples with different thicknesses of the buffer $(d)$ is needed to make this conclusion a general one.

\section{Acknowledgments}

The authors are thankful to Dr. W. Mac and Dr. K. Szadkowski for their help in data acquisition. This work was supported by the Committee for Scientific Research (Poland) grant no. 7T08C01709.

\section{References}

[1] M. Grynberg, S. Huant, G. Martinez, J. Kossut, T. Wojtowicz, G. Karczewski, J.M. Shi, F.M. Peeters, J.T. Devreese, Phys. Rev. B 54, 1467 (1996).

[2] K. Seeger, Semiconductor Physics, Springer-Verlag, Wien 1973.

[3] N.F. Mott, Philos. Mag, 19; 835 (1969).

[4] B.I. Shklovskii, A.L. Efros, Electronic Properties of Doped Semiconductors, Springer, Berlin 1984.

[5] B.I. Shklovskii, B.Z. Spivak, in: Hopping Transport in Solids, Eds. M. Pollak, B. Shklovskii, North-Holland, Amsterdam 1991, p. 271.

[6] G. Bergmann, Phys. Rep. 107, 1 (1984).

[7] B.I. Shklovskii, Pis'ma Zh. Eksp. Teor. Fiz. 36, 43 (1982). 